\title{
A RESULT ON MULTIDIMENSIONAL QUANTIZATION
}

\author{
ERIC B. HALL AND GARY L. WISE \\ (Communicated by George C. Papanicolaou)
}

\begin{abstract}
For any integer $N>1$, a probability space, a Gaussian random vector $X$ defined on the space with a positive definite covariance matrix, and an $N$-level quantizer $Q$ are presented such that the random vector $Q(X)$ takes on each of the $N$ values in its range with equal probability and such that $X$ and $Q(X)$ are independent.
\end{abstract}

\section{INTRODUCTION}

Quantization, the process by which a set is mapped into a finite subset of a given cardinality, plays a pivotal role in virtually any application that requires analog to digital conversion; indeed, it is at the heart of much of modern digital technology. In such applications, a quantizer is often taken to be a function mapping $\mathbf{R}^{k}$ into a subset of $\mathbf{R}^{k}$ of cardinality $N$, where $k$ is a positive integer and $N$ is an integer greater than one (see, e.g., $[1,5,3,6,2,7,9])$. In this paper we present what might be a surprising consequence of such a general approach to quantization.

\section{DEVELOPMENT}

For a topological space $T$, we will let $\mathscr{B}(T)$ denote the family of Borel subsets of $T$. For a set $S$, we will let $\mathbf{P}(S)$ denote the power set of $S$ and $I_{S}$ denote the indicator function of $S$. By a standard Gaussian measure we will mean a Gaussian measure whose first moment is zero and whose second moment is one. Let $k$ be a positive integer. For any measure $m$ on $\left(\mathbf{R}^{k}, \mathscr{B}\left(\mathbf{R}^{k}\right)\right)$ we will let $m_{*}$ denote the inner measure on $\left(\mathbf{R}^{k}, \mathbf{P}\left(\mathbf{R}^{k}\right)\right)$ induced by $m$ and we will let $m^{*}$ denote the outer measure on $\left(\mathbf{R}^{k}, \mathbf{P}\left(\mathbf{R}^{k}\right)\right)$ induced by $m$. Recall from [4, p. 61] that if $B \in \mathscr{B}\left(\mathbf{R}^{k}\right)$ and $A \in \mathbf{P}\left(\mathbf{R}^{k}\right)$, then $m_{*}(B \cap A)+$ $m^{*}\left(B \cap A^{c}\right)=m(B)$. We will let $\lambda$ denote Lebesgue measure on $(\mathbf{R}, \mathscr{B}(\mathbf{R}))$ and $\Lambda$ denote Lebesgue measure on $\left(\mathbf{R}^{k}, \mathscr{B}\left(\mathbf{R}^{k}\right)\right)$ for integers $k>1$, where $k$ will be determined from the context. Recall that for a measure space

Received by the editors April 20, 1991 and, in revised form, September 17, 1991; a preliminary version of this paper was presented at the 863rd meeting of the American Mathematical Society.

1991 Mathematics Subject Classification. Primary 60A10; Secondary 62H12.

Key words and phrases. Multidimensional quantization, saturated nonmeasurable sets.

The first author was supported by the University Research Council of Southern Methodist University. The second author was supported by the Office of Naval Research under Grant N0001490-J-1712. 
$\left(\mathbf{R}^{k}, \mathscr{B}\left(\mathbf{R}^{k}\right), m\right)$, a subset $S$ of $\mathbf{R}^{k}$ is said to be a saturated non- $m$-measurable set if $m_{*}(S)=m_{*}\left(S^{c}\right)=0$. Finally, a $k$-dimensional quantizer of a random variable $X$ defined on a probability space $(\Omega, \mathscr{F}, P)$ is any function $Q: \mathbf{R}^{k} \rightarrow F$ such that $F$ is a finite subset of $\mathbf{R}^{k}$, such that $Q(x)=x$ for all $x$ in $F$ (i.e., such that $Q$ restricted to $F$ is the identity map on $F$ ) and such that $Q(X)$ is itself a random variable defined on $(\Omega, \mathscr{F}, P)$. If $F$ is a finite subset of $\mathbf{R}^{k}$ with cardinality $N$ then a quantizer $Q: \mathbf{R}^{k} \rightarrow F$ of a random variable $X$ is said to be an $N$-level quantizer.

The following lemma is proved in [8, pp. 381-382].

Lemma 1. For any positive integer $M$ there exist $M$ disjoint subsets $Z_{1}, Z_{2}$, $\ldots, Z_{M}$ of the real line such that $Z_{1}, Z_{2}, \ldots, Z_{M}$ and $Z=Z_{1} \cup \cdots \cup Z_{M}$ are saturated non- $\lambda$-measurable sets.

The next result is an immediate consequence of Lemma 1.

Corollary 1. For any integer $N>1$ there exist $N$ subsets $T_{1}, T_{2}, \ldots, T_{N}$ of the real line that partition the real line and are such that for each positive integer $j \leq N, T_{j}$ is a saturated non- $\lambda$-measurable set.

For our purposes the following corollary will prove useful.

Corollary 2. For any positive integer $k$ and any integer $N>1$, there exist $N$ subsets $S_{1}, S_{2}, \ldots, S_{N}$ of $\mathbf{R}^{k}$ that partition $\mathbf{R}^{k}$ and are such that, for each positive integer $j \leq N, S_{j}$ is a saturated non- $\Lambda$-measurable set.

Proof. For $k=1$, the result follows from Corollary 1 . Assume $k>1$. Let $T_{1}, \ldots, T_{N}$ be a partition of the real line as given by Corollary 1 . For positive integers $j \leq N$, let $S_{j}=T_{j} \times \mathbf{R} \times \cdots \times \mathbf{R} \subset \mathbf{R}^{k}$. Fix a positive integer $j \leq N$ and assume that there exists an $\mathscr{F}_{\sigma}$ subset $B$ of $\mathbf{R}^{k}$ such that $B \subset S_{j}$ and $\Lambda(B)>0$. Define a subset $\widehat{B}$ of $\mathbf{R}$ as follows:

$$
\widehat{B}=\left\{b_{1} \in \mathbf{R}:\left(b_{1}, b_{2}, \ldots, b_{k}\right) \in B \text { for some }\left(b_{2}, \ldots, b_{k}\right) \in \mathbf{R}^{k-1}\right\} .
$$

Note that $\widehat{B} \in \mathscr{B}(\mathbf{R})$. Further, notice that $\lambda(\widehat{B})>0$ since $B \subset \widehat{B} \times \mathbf{R} \times \cdots \times \mathbf{R} \subset$ $\mathbf{R}^{k}$ and $\Lambda(B)>0$. But, $\lambda(\widehat{B})=0$ since $\widehat{B} \subset T_{j}$ and $\lambda_{*}\left(T_{j}\right)=0$. This contradiction implies that $\Lambda(B)=0$ and hence that $\Lambda_{*}\left(S_{j}\right)=0$. It follows similarly that $\Lambda_{*}\left(S_{j}^{c}\right)=0$ aiso. Q.E.D.

Lemma 2. For a positive integer $k$ and an integer $N>1$, let $S_{1}, S_{2}, \ldots, S_{N}$ comprise a partition of $\mathbf{R}^{k}$ such that for each positive integer $j \leq N, S_{j}$ is a saturated non- $\Lambda$-measurable set. The set

$$
\mathscr{F}=\left\{\left(S_{1} \cap A_{1}\right) \cup \cdots \cup\left(S_{N} \cap A_{N}\right): A_{i} \in \mathscr{B}\left(\mathbf{R}^{k}\right) \text { for } 1 \leq i \leq N\right\}
$$

is a $\sigma$-algebra on $\mathbf{R}^{k}$.

Proof. Choosing $A_{1}=\cdots=A_{N}=\varnothing$ implies that $\varnothing \in \mathscr{F}$. Let $A$ be an element of $\mathscr{F}$. Then $A=\left(S_{1} \cap A_{1}\right) \cup \cdots \cup\left(S_{N} \cap A_{N}\right)$ for some choice of the $A_{i}$ 's from $\mathscr{B}\left(\mathbf{R}^{k}\right)$. Further, $A^{c}=\left(S_{1} \cap A_{1}\right)^{c} \cap \cdots \cap\left(S_{N} \cap A_{N}\right)^{C}$. Since

$$
S_{i}^{c}=\bigcup_{j=1}^{N} S_{(i \neq j)},
$$


it follows that

$$
A^{c}=\bigcap_{i=1}^{N} \bigcup_{j=1}^{N} S_{j} \cup A_{i}^{c}
$$

Hence $A^{c}$ is a finite union of sets, each of which is of one of the following three forms:

(i) $S_{n_{1}} \cap \cdots \cap S_{n_{k}} \cap B$ where $1 \leq n_{1}<\cdots<n_{k} \leq N, k>1$, and $B \in \mathscr{B}\left(\mathbf{R}^{k}\right)$;

(ii) $S_{j} \cap B$ for $1 \leq j \leq N$ and $B \in \mathscr{B}\left(\mathbf{R}^{k}\right)$;

(iii) $B \in \mathscr{B}\left(\mathbf{R}^{k}\right)$.

Every set of the form given by (i) is empty since the $S_{i}$ 's are disjoint. Further, any set $B \in \mathscr{B}\left(\mathbf{R}^{k}\right)$ may be expressed as $B=\left(S_{1} \cap B\right) \cup \cdots \cup\left(S_{N} \cap B\right)$. Hence, $A^{c}$ is an element of $\mathscr{F}$.

Finally, if $B_{1}, B_{2}, \ldots$ are in $\mathscr{F}$, then for some choice of the $A_{i, j}$ 's from $\mathscr{B}\left(\mathbf{R}^{k}\right)$,

$$
\bigcup_{i=1}^{\infty} B_{i}=\bigcup_{i=1}^{\infty} \bigcap_{j=1}^{N}\left(S_{j} \cap A_{i, j}\right)=\bigcap_{j=1}^{N} S_{j} \cap\left(\bigcup_{i=1}^{\infty} A_{i, j}\right) \in \mathscr{F} \text {. Q.E.D. }
$$

Recall that two measures $P_{1}$ and $P_{2}$ on a given measurable space $(\Omega, \mathscr{G})$ are said to be equivalent if $\left\{A \in \mathscr{G}: P_{1}(A)=0\right\}=\left\{A \in \mathscr{G}: P_{2}(A)=0\right\}$. Notice that for sets $S_{1}, S_{2}, \ldots, S_{N}$ as above, it follows that, for any positive integer $i \leq N$ and any $\mathscr{B}\left(\mathbf{R}^{k}\right)$-measurable set $H, P_{*}\left(S_{i} \cap H\right)=0, P_{*}\left(S_{i}^{c} \cap H\right)=0$, $P^{*}\left(S_{i} \cap H\right)=P(H)$, and $P^{*}\left(S_{i}^{c} \cap H\right)=P(H)$ for any probability measure $P$ on $\left(\mathbf{R}^{k}, \mathscr{B}\left(\mathbf{R}^{k}\right)\right)$ that is equivalent to Lebesgue measure on $\left(\mathbf{R}^{k}, \mathscr{B}\left(\mathbf{R}^{k}\right)\right)$. The following lemma will be used in the proof of a subsequent theorem.

Lemma 3. For a positive integer $k$ and an integer $N>1$, let $S_{1}, S_{2}, \ldots, S_{N}$ comprise a partition of $\mathbf{R}^{k}$ such that for each positive integer $j \leq N, S_{j}$ is a saturated non- $\Lambda$-measurable set. Let $P$ be a probability measure on $\left(\mathbf{R}^{k}, \mathscr{B}\left(\mathbf{R}^{k}\right)\right)$ that is equivalent to Lebesgue measure on $\left(\mathbf{R}^{k}, \mathscr{B}\left(\mathbf{R}^{k}\right)\right)$. Let $A_{1}, \ldots, A_{N}$ and $B_{1}, \ldots, B_{N}$ be sets from $\mathscr{B}\left(\mathbf{R}^{k}\right)$ such that

$$
\left(S_{1} \cap A_{1}\right) \cup \cdots \cup\left(S_{N} \cap A_{N}\right)=\left(S_{1} \cap B_{1}\right) \cup \cdots \cup\left(S_{N} \cap B_{N}\right) .
$$

Then $P\left(A_{i} \triangle B_{i}\right)=0$ for any positive integer $i \leq N$ where for any two subsets $A$ and $B$ of $\mathbf{R}^{k}, A \triangle B$ denotes the symmetric difference of $A$ and $B$.

Proof. Fix a positive integer $i \leq N$. By assumption,

$$
\left(S_{1} \cap A_{1}\right) \cup \cdots \cup\left(S_{N} \cap A_{N}\right)=\left(S_{1} \cap B_{1}\right) \cup \cdots \cup\left(S_{N} \cap B_{N}\right) .
$$

Intersecting each side with $S_{i}$ implies that $\left(S_{i} \cap A_{i}\right)=\left(S_{i} \cap B_{i}\right)$, which implies that $\left(S_{i} \cap A_{i}\right) \cap\left(S_{i} \cap B_{i}\right)^{c}=\left(S_{i} \cap A_{i}\right) \cap\left(S_{i}^{c} \cup B_{i}^{c}\right)=\left(S_{i} \cap A_{i} \cap S_{i}^{c}\right) \cup\left(S_{i} \cap A_{i} \cap B_{i}^{c}\right)=$ $\left(S_{i} \cap A_{i} \cap B_{i}^{c}\right)=\varnothing$ and, similarly, that $\left(S_{i} \cap B_{i} \cap A_{i}^{c}\right)=\varnothing$. Thus, we see that $\left(S_{i} \cap A_{i} \cap B_{i}^{c}\right) \cup\left(S_{i} \cap B_{i} \cap A_{i}^{c}\right)=S_{i} \cap\left(A_{i} \triangle B_{i}\right)=\varnothing$. Since $\left(A_{i} \triangle B_{i}\right) \in \mathscr{B}\left(\mathbf{R}^{k}\right)$, it follows that $P\left(A_{i} \triangle B_{i}\right)=P^{*}\left(S_{i} \cap\left(A_{i} \triangle B_{i}\right)\right)=P^{*}(\varnothing)=0$. Q.E.D.

The following theorem provides a probability space upon which the principal result of this paper will be based.

Theorem 1. For a positive integer $k$ and an integer $N>1$, let $S_{1}, S_{2}, \ldots, S_{N}$ comprise a partition of $\mathbf{R}^{k}$ such that for each positive integer $j \leq N, S_{j}$ is a saturated non- $\Lambda$-measurable set. Let $P$ be a probability measure on $\left(\mathbf{R}^{k}, \mathscr{B}\left(\mathbf{R}^{k}\right)\right)$ 
that is equivalent to Lebesgue measure on $\left(\mathbf{R}^{k}, \mathscr{B}\left(\mathbf{R}^{k}\right)\right)$. There exists a probability space $\left(\mathbf{R}^{k}, \mathscr{G}, \mu\right)$ such that $\mathscr{G}$ includes $\mathscr{B}\left(\mathbf{R}^{k}\right)$, such that $\mathscr{G}$ contains $S_{1}, \ldots, S_{N}$, such that the measure $\mu$ agrees with $P$ on $\mathscr{B}\left(\mathbf{R}^{k}\right)$, and such that $\mathscr{B}\left(\mathbf{R}^{k}\right)$ is independent of $\sigma\left(S_{1}, \ldots, S_{N}\right)$.

Proof. Let $\mathscr{G}$ be the $\sigma$-algebra $\mathscr{F}$ provided by Lemma 2. Recall that $\mathscr{F}$ contains all sets of the form $\left(S_{1} \cap A_{1}\right) \cup \cdots \cup\left(S_{n} \cap A_{n}\right)$ where $A_{i} \in \mathscr{B}\left(\mathbf{R}^{k}\right)$ for each positive integer $i \leq N$. If $A \in \mathscr{B}\left(\mathbf{R}^{k}\right)$ then choosing $A_{1}=\cdots=A_{N}=A$ implies that $A \in \mathscr{G}$. Similarly, for any positive integer $i \leq N$, setting $A_{i}=\mathbf{R}^{k}$ and all other $A_{j}$ 's equal to the empty set implies that $S_{i} \in \mathscr{G}$. Define a measure $\mu$ on the measurable space $\left(\mathbf{R}^{k}, \mathscr{G}\right)$ via

$$
\mu\left(\left(S_{1} \cap A_{1}\right) \cup \cdots \cup\left(S_{N} \cap A_{N}\right)\right)=\frac{1}{N}\left(P\left(A_{1}\right)+\cdots+P\left(A_{N}\right)\right)
$$

for $\left(S_{1} \cap A_{1}\right) \cup \cdots \cup\left(S_{N} \cap A_{N}\right) \in \mathscr{G}$. That $\mu$ is well defined follows from Lemma 3 and that $\mu$ is in fact a probability measure that agrees with $P$ on $\mathscr{B}\left(\mathbf{R}^{k}\right)$ is then straightforward. Further notice that $\mu\left(S_{i}\right)=1 / N$ for each positive integer $i \leq N$ and that, for any set $B \in \mathscr{B}\left(\mathbf{R}^{k}\right)$ and any positive integer $i \leq N, \mu\left(S_{i} \cap B\right)=\frac{1}{N} P(B)=\mu\left(S_{i}\right) \mu(B)$. Thus $S_{i}$ is independent of $\mathscr{B}\left(\mathbf{R}^{k}\right)$ for each positive integer $i \leq N$. Finally, notice that $\mathscr{B}\left(\mathbf{R}^{k}\right)$ is in fact independent of $\sigma\left(S_{1}, \ldots, S_{N}\right)$ since $\left\{\varnothing, S_{1}, \ldots, S_{N}\right\}$ is a $\pi$-system. Q.E.D.

We are now in a position to state and prove the principal result of this paper.

Theorem 2. Let $k$ be a positive integer and let $N$ be an integer greater than one. There exists a probability space $(\Omega, \mathscr{S}, \nu)$, a Gaussian random vector $X$ defined on $(\Omega, \mathscr{S}, \nu)$ taking values in $\mathbf{R}^{k}$ with a positive definite covariance matrix, and an $N$-level $k$-dimensional quantizer $Q: \mathbf{R}^{k} \rightarrow F$ such that $\nu(Q(X)=x)=1 / N$ for each $x$ in $F$ and such that $X$ and $Q(X)$ are independent.

Proof. Let $S_{1}, \ldots, S_{N}$ be sets as provided by Corollary 2. For these $N$ subsets of $\mathbf{R}^{k}$, let $(\Omega, \mathscr{S}, \nu)$ be a probability space as provided by Theorem 1 where $P$ is chosen to be the product measure induced by placing standard Gaussian measure on each factor of $\left(\mathbf{R}^{k}, \mathscr{B}\left(\mathbf{R}^{k}\right)\right)$. For each positive integer $i \leq N$, let $\alpha_{i}$ be an element from $S_{i}$. Let $F$ denote the set $\left\{\alpha_{1}, \ldots, \alpha_{N}\right\}$. Define an $N$-level $k$-dimensional quantizer $Q: \mathbf{R}^{k} \rightarrow F$ via $Q(x)=\sum_{i=1}^{N} \alpha_{i} I_{S_{i}}(x)$. Further, notice that the random vector $X(\omega)=\omega ; \omega \in \Omega$, is a zero mean Gaussian random vector defined on $(\Omega, \mathscr{S}, \nu)$ whose covariance matrix is the $k \times k$ identity matrix. Also, notice that for $1 \leq i \leq N, \nu\left(Q(X(\omega))=\alpha_{i}\right)=$ $\nu\left(\omega \in S_{i}\right)=1 / N$. Finally, notice that $X$ and $Q(X)$ are independent via Theorem 1. Q.E.D.

\section{REFERENCES}

1. A. Gersho, Asymptotically optimal block quantization, IEEE Trans. Inform. Theory IT-25 (1979), 373-380.

2. R. M. Gray and E. D. Karnin, Multiple local optima in vector quantizers, IEEE Trans. Inform. Theory IT-28 (1982), 256-261.

3. R. M. Gray, J. C. Kieffer, and Y. Linde, Locally optimal block quantizer design, Inform. and Control 45 (1980), 178-198.

4. P. R. Halmos, Measure theory, Springer-Verlag, New York, 1974. 
5. Y. Linde, A. Buzo, and R. M. Gray, An algorithm for vector quantizer design, IEEE Trans. Comm. COM-28 (1980), 84-95.

6. D. Pollard, Quantization and the method of k-means, IEEE Trans. Inform. Theory IT-28 (1982), 199-205.

7. M. J. Sabin and R. M. Gray, Global convergence and empirical consistency of the generalized Lloyd algorithm, IEEE Trans. Inform. Theory IT-32 (1986), 148-155.

8. M. Shiffman, Measure-theoretic properties of non-measurable sets, Pacific J. Math. 138 (1989), 357-389.

9. P. F. Swaszek, $A$ vector quantizer for the Laplacian source, IEEE Trans. Inform. Theory 37 (1991), 1355-1365.

Department of Electrical Engineering, Southern Methodist University, Dallas, TEXAS 75275

E-mail address: ebh@smunews.smu.edu

Department of Electrical and Computer Engineering, and Department of Mathematics, The University of TeXas at Austin, Austin, TeXas 78712

E-mail address: gwise@ccwf.cc.utexas.edu 